\title{
A Game-Theoretic Approach for Designing Mixed Mutation Strategies
}

\author{
Jun $\mathrm{He}^{1,2}$ and Xin Yao ${ }^{1}$ \\ 1 School of Computer Science, The University of Birmingham, \\ Edgbaston, Birmingham B15 2TT, U.K. \\ j.he@cs.bham.ac.uk \\ 2 Department of Computer Science, Beijing Jiaotong University, Beijing, China
}

\begin{abstract}
Different mutation operators have been proposed in evolutionary programming. However, each operator may be efficient in solving a subset of problems, but will fail in another one. Through a mixture of various mutation operators, it is possible to integrate their advantages together. This paper presents a game-theoretic approach for designing evolutionary programming with a mixed mutation strategy. The approach is applied to design a mixed strategy using Gaussian and Cauchy mutations. The experimental results show the mixed strategy can obtain the same performance as, or even better than the best of pure strategies.
\end{abstract}

\section{Introduction}

Several mutation operators have been proposed in evolutionary programming (EP), e.g., Gaussian, Cauchy and Lévy mutations [1/2]. According to no free lunch theorem [4], none of mutation operators is efficient in solving all optimization problems, but only in a subset of problems. Experiments show that Gaussian mutation has a good performance for some unimodal functions and multimodal functions with only a few local optimal points; Cauchy mutation works well on multimodal functions with many local optimal points [2].

An improvement to conventional EP is to apply several mutation operators in one algorithm and integrate their advantages together. This idea is not completely new to the community of evolutionary programming. An early implementation is a linear combination of Gaussian and Cauchy distributions [5]. This combination can be viewed a new mutation operator, whose probability distribution is a convolution of Gaussian and Cauchy's probability distributions. IFEP 2] adopts another technique: each individual implements Cauchy and Gaussian mutations simultaneously and generates two individuals; the better one will be chosen in the next generation. In 3, the idea of IFEP is developed further into mixing Lévy distribution with various scaling parameters.

Different from the above work, this paper presents an alternative approach to design a mixed mutation strategy. Inspired from game theory, individuals in EP are regarded as players in a game. Each individual will choose a mutation strategy from its strategy set based on a selection probability, and then generate an offspring by this strategy. 
The central topic of game theory is the interactions and strategies among a group of players 6 6]. Game theory already has a few applications in evolutionary algorithms, mainly in analyzing cooperative co-evolution algorithms [8 910 . This paper concentrates on designing co-evolutionary algorithms, rather than analyzing them.

The rest of this paper is organized as follows: Section 2 introduces EP using mixed strategies; Section 3 illustrates a game-theoretic approach to design mixed strategies; Section 4 describes a case study of mixing Cauchy and Gaussian mutations; Section 5 reports experimental results; Section 6 gives conclusions.

\section{Evolutionary Programming and Mixed Mutation Strategies}

In this paper EP is used to find a minimum $\boldsymbol{x}_{\text {min }}$ of a continuous function $f(\boldsymbol{x})$, that is,

$$
f\left(\boldsymbol{x}_{\min }\right) \leq f(\boldsymbol{x}), \quad \boldsymbol{x} \in D,
$$

where $D$ is a hypercube in $R^{n}, n$ is the dimension. Conventional EP using a single mutation operator can be described as follows [2]:

1. Initialization: Generate an initial population consisting of $\mu$ individuals at random. Each individual is represented a set of real vectors $\left(\boldsymbol{x}_{i}, \boldsymbol{\sigma}_{i}\right)$,

$$
\begin{array}{ll}
\boldsymbol{x}_{i}=\left(x_{i}(1), x_{i}(2), \cdots, x_{i}(n)\right), & i=1, \cdots, \mu \\
\boldsymbol{\sigma}_{i}=\left(\sigma_{i}(1), \sigma_{i}(2), \cdots, \sigma_{i}(n)\right), & i=1, \cdots, \mu .
\end{array}
$$

2. Mutation: For each parent $\left(\boldsymbol{x}_{i}^{(t)}, \boldsymbol{\sigma}_{i}^{(t)}\right)$ (where $t$ represents generation), create an offspring $\left(\boldsymbol{x}_{i}^{\prime}, \boldsymbol{\sigma}_{i}^{\prime}\right)$ as follows:

$$
\begin{aligned}
& \sigma_{i}^{\prime}(j)=\sigma_{i}^{(t)}(j) \exp \left\{\tau N(0,1)+\tau^{\prime} N_{j}(0,1)\right\}, j=1, \cdots, n, \\
& x_{i}^{\prime}(j)=x_{i}^{(t)}(j)+\sigma_{i}^{(t+1)}(j) X_{j}, \quad j=1, \cdots, n,
\end{aligned}
$$

where $N(0,1)$ stands for a Gaussian random variable generated for a given $i, N_{j}(0,1)$ a Gaussian random variable generated for each $j$, and $X_{j}$ is a random variable generated for each $\mathrm{j}$. Controlling parameters $\tau$ and $\tau^{\prime}$ are chosen as the same as in [2].

3. Fitness Evaluation: For $\mu$ parents and their $\mu$ offspring, calculate their fitness value $f_{1}, f_{2}, \cdots, f_{2 \mu}$.

4. Selection: Define and initialize a winning function for every individual in parent and offspring population as $w_{i}=0, i=1,2, \cdots, 2 \mu$. For each individual $i$, select one fitness function, say $f_{j}$ and compare the two fitness functions. If $f_{i}$ is less than $f_{j}$, then let $w_{i}=w_{i}+1$. Perform this procedure $q$ times for each inidivual.

5. Select $\mu$ individuals that have the largest winning values to be parents for the next generation.

6. Repeat step 2-6, until the stopping criteria are satisfied. 
To avoid the step size $\sigma$ falling too low to zero, a lower bound $\sigma_{\text {min }}$ should be put on $\sigma$ [1112. So a revised scheme of updating $\sigma$ is given by:

$$
\sigma_{i}^{\prime}(j)=\left(\sigma_{\min }+\sigma_{i}^{(t)}(j)\right) \exp \left\{\tau N(0,1)+\tau^{\prime} X_{j}\right\} .
$$

where $\sigma_{\min }>0$ is the minimum value of step size $\sigma$.

A mutation operator is called mutation strategy $s$ if the random variable $X_{j}$ in Eq. (2) satisfies the probability distribution function $F_{s}$. A set of mutation strategies consists of Cauchy, Gaussian, Lévy and other probability distributions. The mixed strategy is described as follows: at each generation, an individual chooses one mutation strategy $s$ from its strategy set based on a selection probability $p(s)$. This probability distribution is called a mixed strategy distribution in the game theory.

The key question is to find out a good, if possible an optimal, mixed probability $p(s)$ for every individual. This mixed distribution may be changed over generations.

\section{Game-Theoretic Design and Theoretical Analysis}

According to [7], each game is specified by a set of rules.

First the players and their strategies are specified. In a game consisting of $\nu$ players, players can be denoted by $I=\{1,2, \cdots, \nu\}$. Each player $i$ has a mutation strategy set available to play in the game, which is denoted by $S$ (assume all players use the same strategy set). A strategy is labeled by a positive integer. A single mutation strategy is called a pure strategy in the terms of game theory. A vector of strategies, $s=\left(s_{1}, s_{2}, \cdots, s_{\nu}\right)$ where $s_{i}$ is a strategy used by player $i$, is called a strategy profile.

In this paper, only two-player game is considered, in this case, $\nu=2$. In EP, a population usually consists of more than 2 individuals, so individuals had to be divided into pairs in order to play a two-players game.

Then a payoff is assigned to each strategy. Given a strategy profile $s$, a realvalued number $\pi_{i}(\boldsymbol{s})$ is assigned to each player $i$, which is called the associated payoff to individual $i$. The combined payoff function $\pi$ of the game assigns to each strategy profile $\mathbf{s}$, which is denoted by a vector $\boldsymbol{\pi}(\boldsymbol{s})=\left(\pi_{1}(\boldsymbol{s}), \cdots, \pi_{\nu}(\boldsymbol{s})\right)$ of payoffs.

Thirdly, it is needed to define an output for each strategy. Assume at the $t$-th generation, two players 1 and 2 use strategy $s_{1}$ and $s_{2}$ respectively to play a game. Then an outcome is generated, which is denoted by $o_{1}\left(s_{1}, s_{2}\right)$ and $o_{2}\left(s_{1}, s_{2}\right)$. The payoffs $\pi_{1}\left(s_{1}, s_{2}\right)$ and $\pi_{2}\left(s_{1}, s_{2}\right)$ are dependent on the outcomes $o_{1}\left(s_{1}, s_{2}\right)$ and $o_{2}\left(s_{1}, s_{2}\right)$. In this paper, only symmetric game is considered, so $\pi_{1}\left(s_{1}, s_{2}\right)=$ $\pi_{2}\left(s_{2}, s_{1}\right)$.

At the end, the game will be played in the following order. Individuals in EP are selected two-by-two from the population, play the two-player game in the pair of $(1,2),(3,4), \cdots,(\mu-1, \mu)$ (where $\mu$ is assumed as an even). They play the game simultaneously. The game is iterated, until some stopping criterion is satisfied. 
A mixed strategy for an individual $i$ is defined by a probability distribution $p_{i}(s)$ over its strategy set. It is used to determine the probability of each strategy being applied in the next iteration. It is dependent on the payoffs of strategies. Denote $\boldsymbol{p}=\left(p_{1}, \cdots, p_{\nu}\right)$.

In theory mixed strategies have some potential advantages over pure strategies. This can be seen from the following simple facts.

Firstly, the global performance of some mixed strategy may reach the same performance as the best pure strategy.

Proposition 1. For a pure strategy $s$, let $E\left[f^{(t)} \mid s\right]$ be the mean best fitness outputted at the generation $t$; for a mixed strategy $\boldsymbol{p}^{(0)}, \cdots, \boldsymbol{p}^{(t-1)}$, let $E\left[f^{(t)}\right.$ $\left.\left(\boldsymbol{p}^{(0)}, \cdots, \boldsymbol{p}^{(t-1)}\right)\right]$ be the mean best fitness outputted at the generation $t$. Then there exists some mixed strategy $\boldsymbol{p}^{(0)}, \cdots, \boldsymbol{p}^{(t-1)}$, such that,

$$
\min _{s \in S} E\left[f^{(t)} \mid s\right] \geq E\left[f^{(t)} \mid \boldsymbol{p}^{(0)}, \cdots, \boldsymbol{p}^{(t-1)}\right] .
$$

Proof. The proof is trivial. Assume $s_{*}$ is the best pure strategy, then choose the mixed strategy as follows: all individuals take the best pure strategy $s_{*}$ at any generation, i.e., $p_{i}^{(t)}\left(s_{*}\right)=1$ for all generation $t$.

The second fact reveals another advantage of mixed strategies: mixed strategies can solve more problems than a pure strategy.

Proposition 2. Denote $\mathcal{F}\left(s_{1}\right)$ to be the problem set which can be solved efficiently by strategy $s_{1}, \mathcal{F}\left(s_{2}\right)$ to be the problem set solved efficiently by strategy $s_{2}$, and $\mathcal{F}\left(s_{1}, s_{2}\right)$ the problem set solved efficiently by a mixed strategy consisting of $s_{1}$ and $s_{2}$, then

$$
\mathcal{F}\left(s_{1}\right) \cup \mathcal{F}\left(s_{2}\right) \subset \mathcal{F}\left(s_{1}, s_{2}\right) .
$$

Proof. The proof is straightforward. For any problem in $\mathcal{F}\left(s_{1}\right)$, chose the mixed strategy as: $p\left(s_{1}\right)=1, p\left(s_{2}\right)=0$. For any problem in $\mathcal{F}\left(s_{1}\right)$, choose the mixed strategy as: $p\left(s_{1}\right)=0, p\left(s_{2}\right)=1$.

\section{Case Study: A Mixed Strategy Using Gaussian and Cauchy Mutations}

Two mutation operators are used in the mixed strategy:

- Gaussian Mutation:

$$
x_{i}^{(t+1)}(j)=x_{i}^{(t)}(j)+\sigma_{i}^{(t+1)}(j) N_{j}(0,1),
$$

where $N_{j}(0,1)$ is Gaussian random variable for each component $j$.

- Cauchy Mutation:

$$
x_{i}^{(t+1)}(j)=x_{i}^{(t)}(j)+\sigma_{i}^{(t+1)}(j) C_{j}(0,1),
$$

where $C_{j}(0,1)$ is a Cauchy random variable for each $j$. 
The output of an individual is defined by the distance how far an individual moves during a successful mutation. Let $\boldsymbol{x}_{i}^{(t)}$ be the parent individual, $\boldsymbol{x}_{i}^{(t+1)}$ be its offspring through a mutation strategy, then the output is

$$
o\left(\boldsymbol{x}_{i}^{(t+1)}\right)= \begin{cases}\max _{1 \leq j \leq n}\left\{\left|x_{i}^{(t+1)}(j)-x_{i}^{(t)}(j)\right|\right\}, & \text { if } f\left(\boldsymbol{x}_{i}^{(t+1)}\right)<f\left(\boldsymbol{x}_{i}^{(t)}\right), \\ 0, & \text { otherwise, }\end{cases}
$$

The output of strategy $s_{1}$ is defined by:

$$
o^{(t+1)}\left(s_{1}\right)=\max _{i}\left\{o\left(\boldsymbol{x}_{i}^{(t+1)}\right) ; \boldsymbol{x}_{i}^{(t+1)} \text { is generated by applying strategy } s_{1}\right\} .
$$

If considering the impact of history strategies, the output (8) is amended as follows:

$$
\bar{o}^{(t+1)}\left(s_{1}\right)= \begin{cases}o^{(t+1)}\left(s_{1}\right), & \text { if } o^{(t+1)}\left(s_{1}\right) \geq \alpha \cdot o^{(t)}\left(s_{1}\right), \\ \alpha \cdot o^{(t)}\left(s_{1}\right), & \text { otherwise, }\end{cases}
$$

where $\alpha \in[0,1]$ is a controlling parameter of how much the previous output will kept in memory. $\alpha=0$ means none from historical data; $\alpha=1.0$ means the output is fully determined by the maximum output in history.

Based on the output (9), the payoff of players are defined by

$$
\pi_{1}\left(s_{1}, s_{2}\right)=\frac{o\left(s_{1}\right)}{o\left(s_{2}\right)}, \quad \pi_{2}\left(s_{1}, s_{2}\right)=\frac{o\left(s_{2}\right)}{o\left(s_{1}\right)} .
$$

However there is a danger in Eq. (10): $o\left(s_{2}\right)$ or $o\left(s_{1}\right)$ could be zero. So it is necessary to add a controlling parameter $\beta$ to avoid this extreme case.

$$
\pi_{1}\left(s_{1}, s_{2}\right)= \begin{cases}\beta, & \text { if } o\left(s_{1}\right) / o\left(s_{2}\right) \leq \beta \\ 1 / \beta, & \text { if } o\left(s_{1}\right) / o\left(s_{2}\right) \geq 1 / \beta \\ o\left(s_{1}\right) / o\left(s_{2}\right), & \text { otherwise }\end{cases}
$$

where $\beta \in[0,1]$, where $\beta=0$ allows payoff to be infinity large, and $\beta=1$ means that payoffs of strategies $s_{1}$ and $s_{2}$ both are equal to 1 .

Now assume the strategy profile is $\mathbf{s}=\left(s_{1}, s_{2}\right)$, the mixed probability distribution $p(s)$ can be calculated by the proportion of its payoff among the total payoffs, e.g,:

$$
p_{1}\left(s_{1}\right)=\frac{\pi_{1}\left(s_{1}, s_{2}\right)}{\pi_{1}\left(s_{1}, s_{2}\right)+\pi_{1}\left(s_{1}, s_{2}\right)} .
$$

\section{Experimental Results and Analysis}

The above EP is evaluated on 7 test functions, which was used to test IFEP in 2]. The description of these functions is given in Table 1 Among them, function $f_{1}$ and $f_{2}$ are unimodal functions, $f_{3}$ and $f_{4}$ multimodal functions with many local minima, $f_{5}-f_{7}$ multimodal functions with only a few local minima.

The parameter setup in the mixed EP are taken as the same values as those in [2]. Population size $\mu=100$, tournament size $q=10$, and initial standard 
Table 1. Seven test functions, where the coefficients of $f 5-f 7$ are given in 2 ]

\begin{tabular}{l|c|c}
\hline test functions & domain & $f_{\min }$ \\
\hline$f_{1}=\sum_{i=1}^{30} x_{i}^{2}$ & {$[-100,100]^{30}$} & 0 \\
$f_{2}=\sum_{i=1}^{30}\left|x_{i}\right|+\prod_{i=1}^{30}\left|x_{i}\right|$ & {$[-100,100]^{30}$} & 0 \\
$f_{3}=-20 \exp \left(-0.2 \sqrt{\frac{1}{30} \sum_{i=1}^{30} x_{i}^{2}}\right)$ & {$[-32,32]^{30}$} & 0 \\
& $-\exp \left(\frac{1}{30} \sum_{i=1}^{30} \cos \left(2 \pi x_{i}\right)\right)+20+e$ & \\
$f_{4}=\frac{1}{4000} \sum_{i=1}^{30} x_{i}^{2}-\prod_{i=1}^{30} \cos \left(x_{i} / \sqrt{i}\right)+1$ & {$[-600,600]^{30}$} & 0 \\
$f_{5}=-\sum_{i=1}^{5}\left(\sum_{j=1}^{4}\left(x_{j}-a_{i j}\right)^{2}+c_{i}\right)^{-1}$ & {$[0,10]^{4}$} & -10.15 \\
$f_{6}=-\sum_{i=1}^{7}\left(\sum_{j=1}^{4}\left(x_{j}-a_{i j}\right)^{2}+c_{i}\right)^{-1}$ & {$[0,10]^{4}$} & -10.34 \\
$f_{7}=-\sum_{i=1}^{10}\left(\sum_{j=1}^{4}\left(x_{j}-a_{i j}\right)^{2}+c_{i}\right)$ & {$[0,10]^{4}$} & -10.54 \\
\hline
\end{tabular}

Table 2. Comparison of mean best fitness between MEP and IFEP, FEP, CEP

\begin{tabular}{c|c|c|c|c|c}
\hline & $\begin{array}{c}\text { function } \\
\text { evaluation }\end{array}$ & $\begin{array}{c}\text { MEP } \\
\text { mean best }\end{array}$ & $\begin{array}{c}\text { IFEP [2] } \\
\text { mean best }\end{array}$ & $\begin{array}{c}\text { FEP [2] } \\
\text { mean best }\end{array}$ & $\begin{array}{c}\text { CEP [2] } \\
\text { mean best }\end{array}$ \\
\hline$f_{1}$ & 150,000 & $9.151 \mathrm{e}-06$ & $4.16 \mathrm{e}-5$ & $5.72 \mathrm{e}-4$ & $1.91 \mathrm{e}-4$ \\
$f_{2}$ & 200,000 & $1.269 \mathrm{e}-03$ & $2.44 \mathrm{e}-2$ & $7.60 \mathrm{e}-2$ & $2.29 \mathrm{e}-2$ \\
$f_{3}$ & 150,000 & $6.590 \mathrm{e}-04$ & $4.83 \mathrm{e}-3$ & $1.76 \mathrm{e}-2$ & 8.79 \\
$f_{4}$ & 200,000 & $1.706 \mathrm{e}-02$ & $4.54 \mathrm{e}-2$ & $2.49 \mathrm{e}-2$ & $8.13 \mathrm{e}-2$ \\
$f_{5}$ & 10,000 & $-8.774 \mathrm{e}+00$ & -6.46 & -5.50 & -6.43 \\
$f_{6}$ & 10,000 & $-9.735 \mathrm{e}+00$ & -7.10 & 5.73 & 7.62 \\
$f_{7}$ & 10,000 & $-9.841 \mathrm{e}+00$ & -7.80 & 6.41 & 8.86 \\
\hline
\end{tabular}

deviation is take as $\sigma=3.0$. The stopping criteria is to stop running at 1500 generations for functions $f_{1}$ and $f_{3}, 2000$ generations for $f_{2}$ and $f_{4}, 100$ generations for $f_{5}-f_{7}$. The lower-bound used in this paper is $\sigma_{\min }=10^{-5}$ for all functions except $f_{4}$. Since $f_{4}$ has a larger definition domain than others, $\sigma_{\min }$ is taken a bigger value $10^{-4}$. Parameters $\alpha$ in Eq.919) and $\beta$ in Eq.(11) is chosen to be 0.9 and 0.05 respectively. At the initial step, the mixed strategy distribution is taken as $(0.5,0.5)$. Results for $f_{1}-f_{4}$ are averaged over 50 independent runs, for $f_{5}-f_{7}$ over 1000 independent runs.

In the following, CEP is EP using Gaussian mutation; FEP using Cauchy mutation; IFEP using Gaussian and Cauchy mutations simultaneously; MEP is the mixed mutation given in this paper.

The first experiment aims to compare MEP with IFEP, FEP and CEP. Table 2 gives results generated by MEP against existing results of IFEP, FEP and CEP from 2. It is observed that MEP is obviously much better than IFEP, $\mathrm{CEP}$ and FEP over all test functions. However, to be honest, this improvement is partly due to the well-chosen low bound $\sigma_{\min }$.

The second experiment intends to compare mixed mutation with Gaussian and Cauchy mutations when they take the same lower bounds. There are three types of results: 
1. In Figures 1, 2, 4 and 6, the mixed strategy can reach the same fitness level as the best pure strategy does.

2. In Figure 2, the mixed strategy has a better performance.

3. In Figure 5, the mixed strategy is a little worse than the best pure strategy (Gaussian), however, the difference is very small.

The third experiment describes the dynamics of the mixed strategy. There are two types of dynamics:

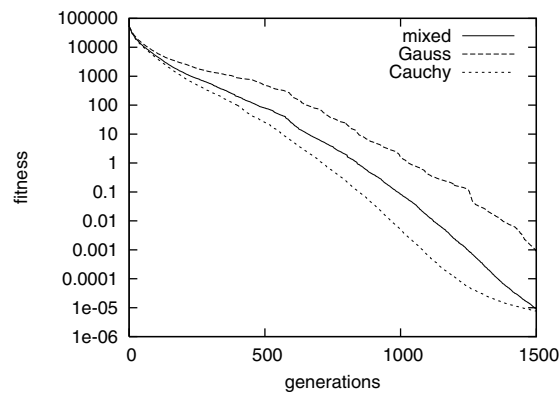

Fig. 1. Function $f_{1}$

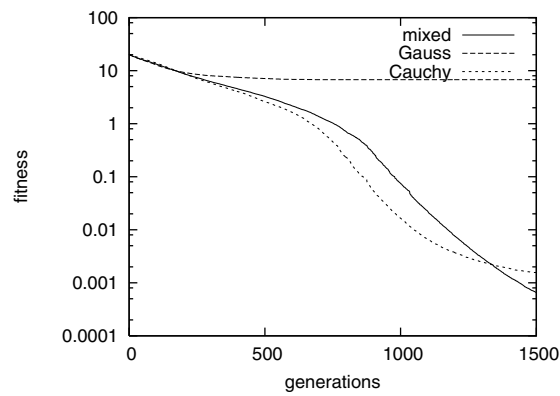

Fig. 3. Function $f_{3}$

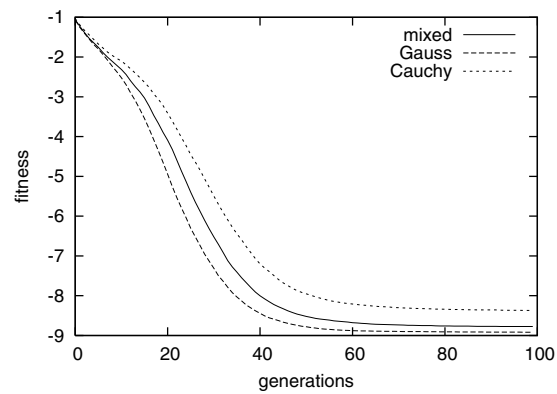

Fig. 5. Function $f_{5}$

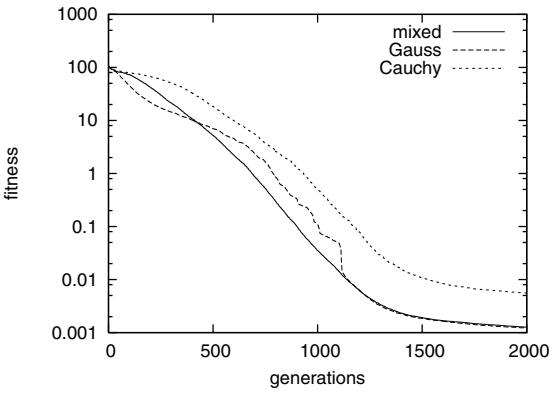

Fig. 2. Function $f_{2}$

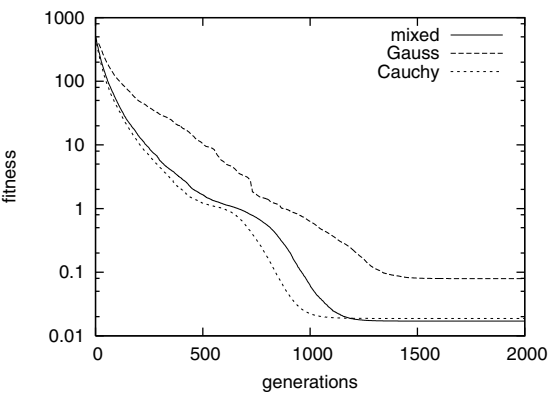

Fig. 4. Function $f_{4}$

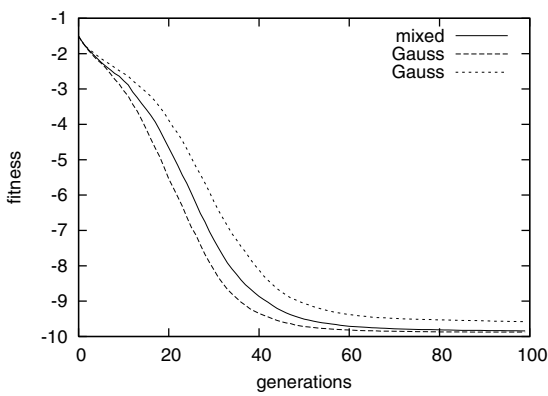

Fig. 6. Function $f_{6}$ 
1. In Figures 7, 10, Cauchy mutation takes a higher percentage as a global search at the early search phase; and then Gaussian mutation holds a dominant position as a local search.

2. Figures 11 and 12 demonstrate another dynamics of the mixed strategy. The number of individuals using Gaussian mutation is almost the same as that of using Cauchy mutation.

The last experiment studies the impact of parameters $\alpha$ and $\beta$ on the performance. The parameter $\alpha$ is regraded as a memory of history. From Table 3, it

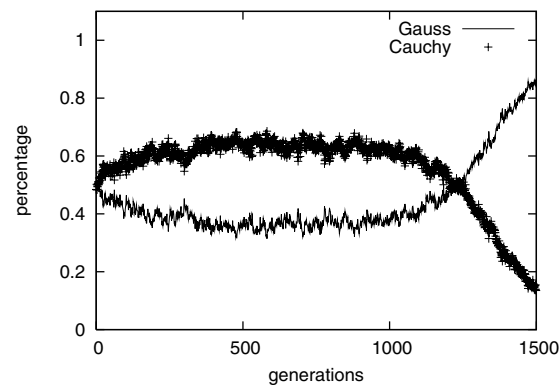

Fig. 7. Function $f_{1}$

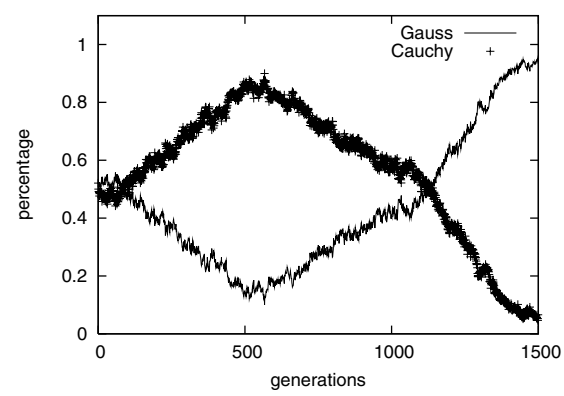

Fig. 9. Function $f_{3}$

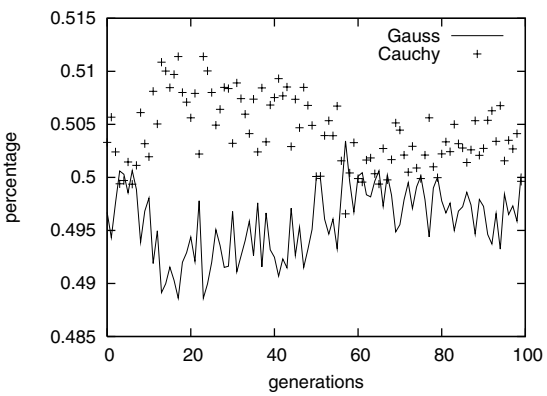

Fig. 11. Function $f_{5}$

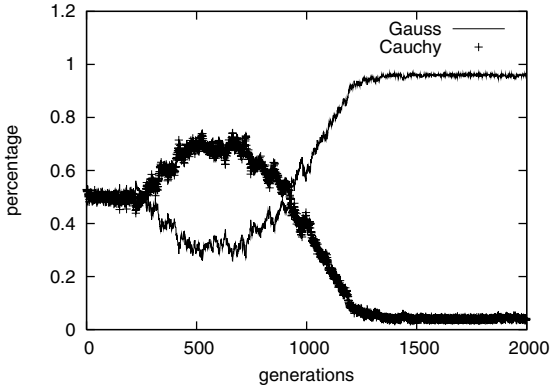

Fig. 8. Function $f_{2}$

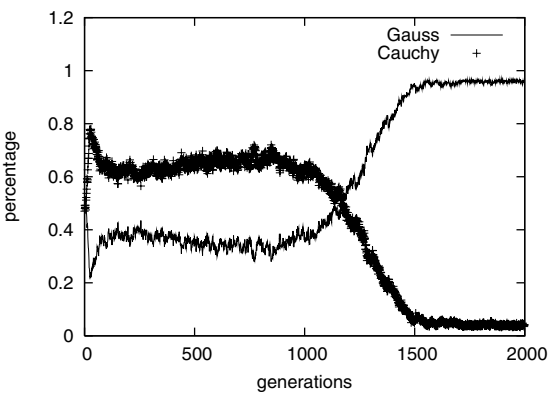

Fig. 10. Function $f_{4}$

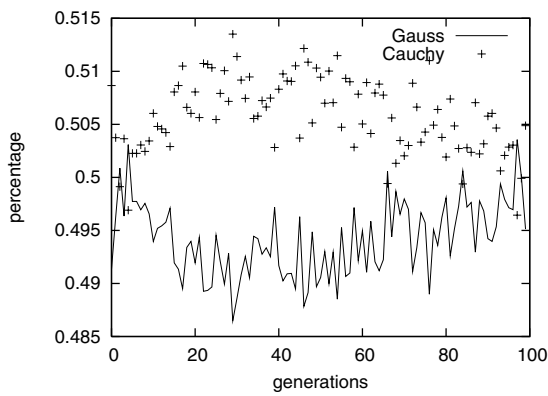

Fig. 12. Function $f_{6}$ 
Table 3. Impact of parameter $\alpha$

\begin{tabular}{c|c|c|c|c|c}
\hline & $\begin{array}{c}\text { function } \\
\text { evaluation }\end{array}$ & $\begin{array}{c}\alpha=0.0 \\
\text { mean best }\end{array}$ & $\begin{array}{c}\alpha=0.5 \\
\text { mean best }\end{array}$ & $\begin{array}{c}\alpha=0.9 \\
\text { mean best }\end{array}$ & $\begin{array}{c}\alpha=1.0 \\
\text { mean best }\end{array}$ \\
\hline$f_{1}$ & 150,000 & $3.342 \mathrm{e}-05$ & $8.461 \mathrm{e}-06$ & $9.151 \mathrm{e}-06$ & $1.974 \mathrm{e}-05$ \\
$f_{2}$ & 200,000 & $1.224 \mathrm{e}-03$ & $1.249 \mathrm{e}-03$ & $1.269 \mathrm{e}-03$ & $1.760 \mathrm{e}-03$ \\
$f_{3}$ & 150,000 & $5.720 \mathrm{e}-04$ & $5.757 \mathrm{e}-04$ & $6.590 \mathrm{e}-04$ & $1.464 \mathrm{e}-03$ \\
$f_{4}$ & 200,000 & $2.967 \mathrm{e}-02$ & $2.761 \mathrm{e}-02$ & $1.706 \mathrm{e}-02$ & $2.123 \mathrm{e}-02$ \\
$f_{5}$ & 10,000 & $-8.478 \mathrm{e}+00$ & $-8.627 \mathrm{e}+00$ & $-8.774 \mathrm{e}+00$ & $-8.685 \mathrm{e}+00$ \\
$f_{6}$ & 10,000 & $-9.539 \mathrm{e}+00$ & $-9.607 \mathrm{e}+00$ & $-9.735 \mathrm{e}+00$ & $-9.706 \mathrm{e}+00$ \\
$f_{7}$ & 10,000 & $-9.814 \mathrm{e}+00$ & $-9.801 \mathrm{e}+00$ & $-9.841 \mathrm{e}+00$ & $-9.658 \mathrm{e}+00$ \\
\hline
\end{tabular}

Table 4. Impact of parameter $\beta$

\begin{tabular}{c|c|c|c|c|c}
\hline & $\begin{array}{c}\text { function } \\
\text { evaluation }\end{array}$ & $\begin{array}{c}\beta=0.0 \\
\text { mean best }\end{array}$ & $\begin{array}{c}\beta=0.05 \\
\text { mean best }\end{array}$ & $\begin{array}{c}\beta=0.5 \\
\text { mean best }\end{array}$ & $\begin{array}{c}\beta=1.0 \\
\text { mean best }\end{array}$ \\
\hline$f_{1}$ & 150,000 & $2.696 \mathrm{e}-05$ & $9.151 \mathrm{e}-06$ & $9.945 \mathrm{e}-06$ & $3.864 \mathrm{e}-05$ \\
$f_{2}$ & 200,000 & $1.207 \mathrm{e}-03$ & $1.269 \mathrm{e}-03$ & $1.521 \mathrm{e}-03$ & $3.692 \mathrm{e}-03$ \\
$f_{3}$ & 150,000 & $2.238 \mathrm{e}-01$ & $6.590 \mathrm{e}-04$ & $1.169 \mathrm{e}-03$ & $1.239 \mathrm{e}-03$ \\
$f_{4}$ & 200,000 & $1.884 \mathrm{e}-02$ & $1.706 \mathrm{e}-02$ & $1.918 \mathrm{e}-02$ & $2.147 \mathrm{e}-02$ \\
$f_{5}$ & 10,000 & $-8.692 \mathrm{e}+00$ & $-8.774 \mathrm{e}+00$ & $-8.708 \mathrm{e}+00$ & $-8.689 \mathrm{e}+00$ \\
$f_{6}$ & 10,000 & $-9.625 \mathrm{e}+00$ & $-9.735 \mathrm{e}+00$ & $-9.610 \mathrm{e}+00$ & $-9.622 \mathrm{e}+00$ \\
$f_{7}$ & 10,000 & $-9.776 \mathrm{e}+00$ & $-9.841 \mathrm{e}+00$ & $-9.818 \mathrm{e}+00$ & $-9.786 \mathrm{e}+00$ \\
\hline
\end{tabular}

is seen that for four different values, MEP has produced a similar performance. Parameter $\beta$ is a threshold to control the scale of each strategy's payoff. Table 4 displays a good performance if $\beta=0.05,0.5,1.0$. However, $\beta=0.0$ is a bad choice for function $f_{3}$.

\section{Conclusions}

This paper presents a game-theoretic approach to design EP using mixed strategies. The conventional EP usually apply a single mutation strategy. However according to no free lunch theorem, none of a single mutation operators cannot solve all problems efficiently no matter how powerful it is. So it is expected that a mixture of different mutation strategies will solve more problems efficiently than a single mutation strategy does. This paper has confirmed this point.

In theory it s easy to see that some mixed strategies could perform at least as good as or eve better than the best pure strategy, and may solve more problems efficiently than one pure strategy. Through a case study, the theoretic prediction has been validated. The experimental results given in this paper have demonstrated that the mixed mutation has obtained the same or nearly same performance as the best of Cauchy and Gaussian mutations over all test functions, and even better in some cases. If only a single mutation strategy is applied, neither Gaussian nor Cauchy mutation can solve all 7 test functions efficiently. 
Further research works include: at present, the evaluation of mixed mutation strategies is only implemented on a few benchmark functions, it is necessary to verify it on more problems; the game designed in this paper is a simple two-player game, it is necessary to study more complex game; if more mutation operators were added into the mixed strategy, it would lead to a more powerful mixed strategy.

Acknowledgements. This work is partially supported by Engineering and Physical Research Council under Grant (GR/T10671/01) and National Natural Science Foundation under Grant (60443003).

\section{References}

1. D. Fogel. Evolution Computation: Toward a New Philosophy of Machine Intelligence. IEEE Press, Piscataway, NJ, 1995.

2. X. Yao, Y. Liu, and G. Lin. Evolutionary programming made faster. IEEE Trans. Evolutionary Computation, 3(2):82-102, 1999.

3. C.-Y. Lee and X. Yao. Evolutionary programming using mutations based on the Lévy probability distribution. IEEE Trans. on Evolutionary Computation, 8(2):1$13,2004$.

4. D. H. Wolpert and W. G. Macready. No free lunch theorem for optimization. IEEE Trans. on Evolutionary Computation, 1(1):67-82, 1997.

5. K. Chellapilla. Combining mutation operators in evolutionary programming. IEEE Trans. on Evolutionary Computation, 2(3):91-96, 1998.

6. J. W. Weibull. Evolutionary Game Theory. MIT press, Cambridge, MA, 1995.

7. P. K. Dutta. Strategies and Games. The MIT Press, Cambridge, MA, 1999.

8. S. G. Ficici, O. Melnik, and J. B. Pollack. A game-theoretic investigation of selection methods used in evolutionary algorithms. In Proc. of 2000 Congress on Evolutionary Computation, pages 880 - 887. IEEE Press, 2000.

9. R. P. Wiegand, W. C. Liles, and K. A. De Jong. Analyzing coperative coevolution with evolutionary game theory. In Proc. of 2002 Congress on Evolutionary Computation, pages 1600-1605. IEEE Press, 2002.

10. S. G. Ficici and J. B. Pollack. A game-theoretic memory mechanism for coevolution. In Proc. of 2003 Genetic and Evolutionary Computation Conference, pages 286-297. Springer, 2003.

11. K.-H. Liang, X. Yao, and C. S. Newton. Adapting self-adaptive parameters in evolutionary algorithms. Applied Intellegence, 15(3):171-180, 2001.

12. D. Fogel, G. Fogel, and K. Ohkura. Multiple-vector self-adaptation in evolutionary algorithms. BioSystems, 61:155-162, 2001. 